'Centro de Salud Familiar Cardenal Raúl Silva Henríquez, Av. La Paz 2470 La Serena, Chile. 2Facultad de Medicina, Universidad Católica del Norte, Coquimbo.

Fuente de apoyo financiero: Proyecto de investigación financiado por el Departamento de Salud Pública, Universidad Católica del Norte La institución

tuvo injerencia en el diseño y revisión del estudio, no así en la ejecución

Recibido el 14 de abril de 2018 aceptado el 30 de julio de 2019

Correspondencia a: Jacqueline Flores Águila Facultad de Medicina Universidad Católica del Norte, Sede Coquimbo. Larrondo 1281, Coquimbo. Chile. jfloresa@ucn.cl

\section{Asociación entre programa de intervención educativa integral y control metabólico de usuarios diabéticos tipo 2 insulino requirentes de Centro de Salud Familiar Cardenal Raúl Silva Henríquez, período 2015-2016 Chile}

\author{
DAYNA OLIVARES ${ }^{1}$, JACQUELINE FLORES ${ }^{2}$
}

\section{The effect of an educational intervention on the metabolic control of diabetic patients}

Background: Diabetic patients have a poor adherence to lifestyle changes and a low compliance with medications. Aim: To study the effect of an educational intervention on the metabolic control of patients with diabetes mellitus. Patients and Methods: We studied insulin requiring diabetic patients attended at a primary health care clinic, who were randomly divided in two groups. One group participated in four modules of an educative intervention, carried out in four occasions. The other group did not participate in the educational sessions. Clinical, anthropometric and laboratory variables of the participants were obtained from their medical records, at three and six months after the intervention. Results: We recruited 22 men aged $64 \pm 14$ years and 48 women aged $63 \pm 10$ years. Thirty-six of these received the educational intervention. Eighty four percent were overweight or obese. The proportion of compensated patients, defined as those having a glycosylated hemoglobin between 7 and 9\%, increased significantly after the educational intervention $\left(\chi^{2}=7.9 p<0.01\right.$, odds ratio: $0.2)$. Conclusions: A educational intervention in patients with insulin requiring diabetes mellitus improved the metabolic control of their disease.

(Rev Med Chile 2019; 147: 1024-1028)

Key words: Carbohidrate Metabolism; Diabetes Mellitus; Early Intervention (Education); Glycated Hemoglobin A.

\footnotetext{
L
} a diabetes Mellitus tipo 2 (DM2) es una enfermedad crónica que resulta un problema de Salud Pública y social ${ }^{1,2}$. La DM2 es una enfermedad que por sí sola es poco sintomática, produciendo como consecuencia un diagnóstico tardío y mayor peligro de presentar complicaciones $^{3}$. Estudios revelan que la importancia epidemiológica de la DM2 no depende solo de su prevalencia, sino que de la carga de morbimorta- lidad asociada a estas complicaciones debido al descontrol metabólico, que en el 2016 se adjudica 10,2\% del presupuesto de Salud de Chile ${ }^{4}$. Los modelos no invasivos mas efectivos se centran en educación y autocuidado, tomando como modelo los avances del Dr. E Wagner estos prototipos se basan en la evidencia de que las relaciones continuas de cuidado entre el usuario y el equipo producen mejorías en los resultados clínicos, involucrando 
en una triada a la comunidad y entorno familiar de la persona ${ }^{5}$. El objetivo general del presente estudio fue evaluar la asociación de la intervención de un programa educativo integral y la mejoría en el control metabólico, estado nutricional de los usuarios Diabéticos insulino requirentes de CESFAM CRSH entre julio de 2015-junio de 2016, La Serena, Chile.

\section{Materiales y Métodos}

Se realizó un estudio de tipo analítico, longitudinal. Se consideró como universo al total de usuarios diabéticos insulino requirentes del CESFAM CRSH. La muestra fue integrada por el total de usuarios intervenidos como grupo de estudio (GI) y se seleccionó aleatoriamente un grupo de usuarios con características similares como control (GC), a los cuáles no se les aplicó la intervención educativa. Esta última consistió en 4 módulos, entregados por un equipo multidisciplinario en dos jornadas, no se midió nivel de conocimientos previos y post sesiones. Se evitó hacer modificaciones en el tratamiento farmacológico y nutricional, salvo dos casos extremos que se ajustó la cantidad de insulina. Como criterios de inclusión se consideró participación en todas las sesiones de la Intervención Integral del usuario DM2 Insulinorequierentes; seguimiento y tratamiento de su DM en el mismo CESFAM y pacientes que tras ser informados del estudio deciden participar en el mismo, previa firma de consentimiento informado. Los criterios de exclusión que se aplicaron fueron: registros incompletos de controles metabólicos, usuarios con alteraciones cognitivas que no le permitan participar o comprender los contenidos planteados. La mayor parte de la información se recolectó de fuentes secundarias. El análisis, incluye las variables de cada sujeto previo a la intervención educativa en caso del GI y en el GC previo a intervenciones individuales de los profesionales. Así mismo el tiempo post se fijó para las variables transcurrido 3 a 6 meses después de cada intervención grupal o individual, según disponibilidad de datos en fichas clínicas. Para el análisis de la información se utilizó paquete estadístico SPSS. La información que se recolectó considerando los requisitos éticos de investigación de Ezequiel Emanuel. La investigación fue aprobada por el Comité de Ética de la Facultad de Medicina de la Universidad Católica del Norte, Chile, según resolución 32/2016.

\section{Resultados}

Ingresaron al estudio 70 sujetos, 36 de ellos participantes del programa educativo y $34 \mathrm{co}-$ rrespondientes al grupo denominado control o de comparación. Según se observa en la Tabla 1, del total de participantes, $68,5 \%$ son de género

Tabla 1. Distribución de la población según variables sociodemográficas y clínicas. La Serena, 2016

\begin{tabular}{|c|c|c|}
\hline Variable & $\begin{array}{l}\text { Hombres } \\
n=22\end{array}$ & $\begin{array}{l}\text { Mujeres } \\
n=48\end{array}$ \\
\hline $\begin{array}{l}\text { Biopsicosocial } \\
\text { Edad; años; promedio } \pm \mathrm{DE}\end{array}$ & $64,3 \pm 13,5$ & $63,3 \pm 10,3$ \\
\hline $\begin{array}{l}\text { Años de escolaridad: años; n; \% } \\
\text { Sin escolaridad } \\
\text { Básica } \\
\text { Media } \\
\text { Superior }\end{array}$ & $\begin{array}{c}2(9 \%) \\
15(68 \%) \\
5(31,8 \%) \\
0(0 \%)\end{array}$ & $\begin{array}{c}3(6,2 \%) \\
32(66,6 \%) \\
13(27 \%) \\
0\end{array}$ \\
\hline Peso; Kg; Promedio \pm DE & $80,6 \pm 13,4$ & $75,3 \pm 16,01$ \\
\hline Talla m; Promedio $\pm \mathrm{DE}$ & $1,66 \pm 0,07$ & $1,51 \pm 0,06$ \\
\hline $\mathrm{IMC} \mathrm{kg/ \textrm {m } ^ { 2 }}$ & $28,9 \pm 4,4$ & $32,9 \pm 6,3$ \\
\hline Glicemia en ayuno; mg/dl; promedio $\pm \mathrm{DE}$ & $169,6 \pm 57,19$ & $207,3 \pm 94,7$ \\
\hline Nivel de hemoglobina a1c; \%; Promedio $\pm \mathrm{DE}$ & $9,6 \pm 2,22$ & $9,9 \pm 2,30$ \\
\hline
\end{tabular}

Fuente: Base de Datos de estudio. Elaboración propia. 
Tabla 2. Distribución de la población en estudio según indicadores clínicos previo y posterior a intervención educativa. La Serena, 2016

\begin{tabular}{|lccccc|}
\hline Variables & \multicolumn{2}{c}{$\begin{array}{c}\text { Grupo }(\mathbf{n}=\mathbf{3 6}) \\
\text { Previo a } \\
\text { Programa } \\
\text { Educativo }\end{array}$} & $\begin{array}{c}\text { Posterior a } \\
\text { Programa } \\
\text { Educativo }\end{array}$ & $\begin{array}{c}\text { Control }(\mathbf{n}=\mathbf{3 4 )} \\
\text { Pre } \\
\text { intervenciones } \\
\text { individuales }\end{array}$ & $\begin{array}{c}\text { Post } \\
\text { intervenciones } \\
\text { individuales }\end{array}$ \\
$<7 \%$ & $0(0 \%)$ & $1(2,8 \%)$ & 3 & 8 \\
\hline $7-9 \%$ & $7(19,4 \%)$ & $16(44,4 \%)$ & 18 & 20 \\
$>9 \%$ & $29(80,6 \%)$ & $19(52,8 \%)$ & 13 & 6 \\
\hline Bajo peso & $<18,5 \mathrm{~kg} / \mathrm{m}^{2}$ & $1(2,8 \%)$ & $1(2,8 \%)$ & 1 & 1 \\
\hline Normopeso & $18,5-24,9 \mathrm{~kg} / \mathrm{m}^{2}$ & $8(22,2 \%)$ & $7(19,4 \%)$ & 4 & 5 \\
\hline Sobrepeso & $25,0-29,9 \mathrm{~kg} / \mathrm{m}^{2}$ & $10(27,8 \%)$ & $11(30,6 \%)$ & 12 & 12 \\
\hline Obesidad & $>30,0 \mathrm{~kg} / \mathrm{m}^{2}$ & $17(47,2 \%)$ & $17(47,2 \%)$ & 17 & 16 \\
\hline
\end{tabular}

Fuente: Base de Datos de estudio. Elaboración propia.

femenino, con edades promedio de 63,3 años y 64,3 años para los varones. El 75\% de la población presentaba mal nutrición por exceso (MNE), con promedio de IMC de $28,9 \mathrm{~kg} / \mathrm{m}^{2}$ y $32,9 \mathrm{~kg} / \mathrm{m}^{2}$, en hombres y mujeres respectivamente. El promedio de glicemia en ayunas y HbAlc es inicialmente levemente mayor en mujeres siendo de 207,3 mg/ dl y $9,9 \%$ respectivamente.

La variable estado nutricional, evaluando peso corporal y distribuido según las categorías para el IMC, se mantuvo sin cambios significativos en cada tiempo de evaluación en un mismo grupo, y entre ellos. El análisis bivariado de cada variable fue mediante OR, donde se clasificaron a los pa- cientes de la siguiente manera; compensados $=$ niveles de HbAlc normal e intermedio (7-9\%) y descompensados $=$ niveles de HbA1c elevados $(\geq 9 \%)$. En las variables sociodemográficas de: edad, género, escolaridad y su relación el grado de control metabólico compensado y no compensado, no se encontraron diferencias significativas $\left(\chi^{2}=1,5, p=0,21\right)$. Las figuras 1 y 2 muestran las variables clínicas, las cuales muestran diferencia significativa entre las categorías compensados y descompensados en el GI, previo y post a la intervención educativa para la variable $\mathrm{HbAlc}$, con un $\chi^{2}=5,063 \mathrm{p}=0,02$, siendo menor la cantidad de usuarios que se mantiene en la categoría $>9 \%$

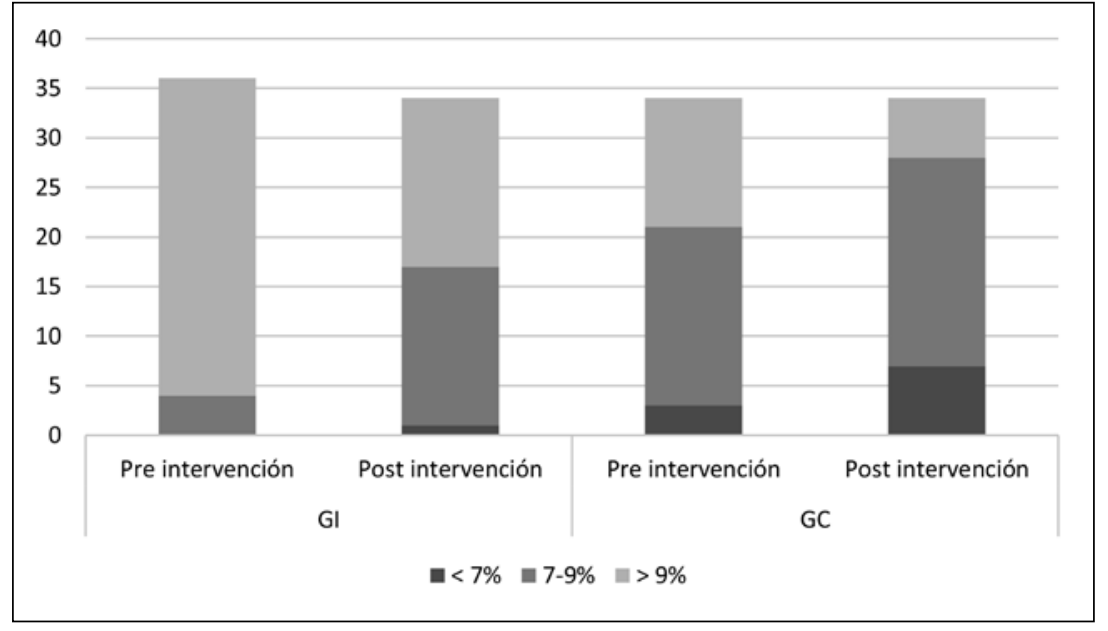

Figura 1. Cambios en el nivel de $\mathrm{HbA} 1 \mathrm{c}$ en dos tiempos de análisis, según participación en programa de intervención educativa. Gl: Grupo de intervención; GC: Grupo control, *Cambio significativo con $p<0,05$ (Prueba $\chi^{2}$ ), Fuente: Base de Datos de estudio. Elaboración propia. 


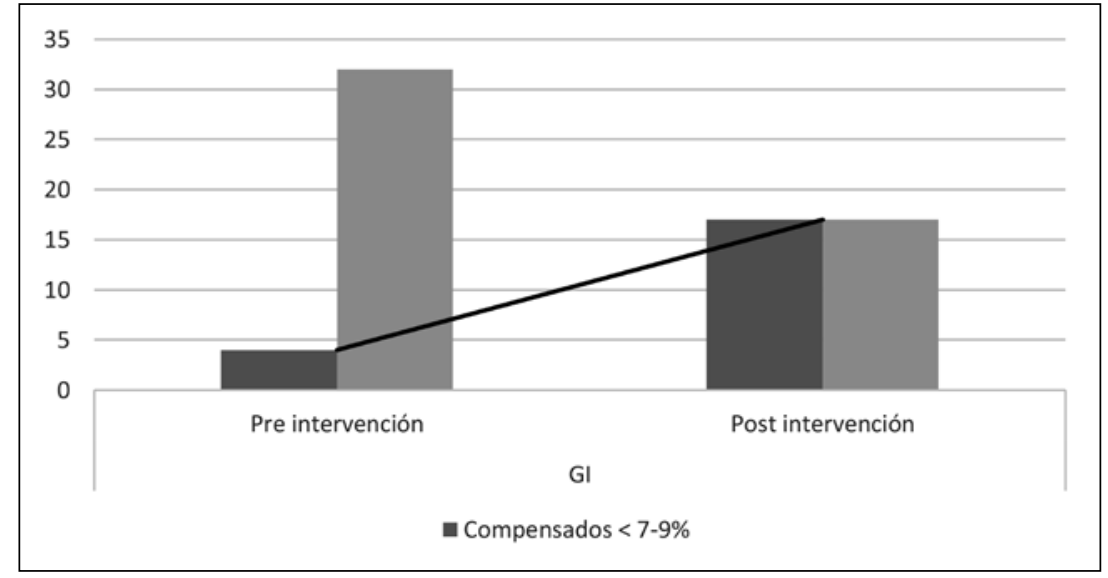

Figura 2. Distribución de la población en estudio según compensación previo y posterior a intervención educativa. Fuente: Base de Datos de estudio. Elaboración propia.
HbA1c. La Figura 2 muestra una asociación positiva en la variable $\mathrm{HbAlc}$ posterior a intervención educativa en grupo de compensados, $\chi^{2}=7,932$ $\mathrm{p}=0,005$, OR: 0,2 siendo la variable intervención educativa factor protector para la compensación, así la chance de presentar mayor compensación en los parámetros clínicos es mayor cuando se expone a la intervención.

\section{Discusión}

El presente estudio tiene implicancias clínicas y epidemiológicas importantes para la práctica clínica y el desarrollo de políticas de salud pública en nuestra realidad local y nacional, puesto que la reducción de la HbAlc se asocia a menor riesgo de complicaciones, por lo que se traduce en menor gasto en la enfermedad a largo plazo con una intervención de bajo costo, como lo es la educación. Los resultados del estudio muestran que la intervención educativa se asocia positivamente en favorecer el logro de metas terapéuticas y mejorar el control glicémico del usuario. El estado Nutricional del usuario evaluado por la variable IMC, presenta un alza, al igual como se presenta en el estudio de Figueroa Suárez M. donde los parámetros bioquímicos disminuyen pero el peso, IMC y colesterol no obtienen cambios significativos en los grupos intervenidos ni en el control ${ }^{12,14}$. De la misma manera que en el estudio de León-Mazón donde se evalúa la eficacia de un programa de educativo en diabetes, se obtienen similares resultados donde se reducen los parámetros pero no se llega a la compensación recomendada $\mathrm{HbA1c}<7 \%$, aún cuando su población en estudio es en promedio menor a la estudiada en la presente investigación, por lo que se debe analizar otros factores que influyen en el control metabólico del usuario ${ }^{15}$. Existe evidencia que apoya el beneficio de la intervención educativa en el control glicémico, pero no es clara en tipos y tiempos de evaluación ${ }^{16,17}$. Se consideran algunas limitaciones del estudio: la presencia de enfermedades concomitantes, bajo n muestral, diferencia en los parámetros clínicos de inicio de ambos grupos analizados, duración de la intervención, número de sesiones es limitada, por lo que podría hacerse un estudio de cohorte para ver resultados en la prevención o retraso de complicaciones crónicas, con intervenciones educativas y evaluaciones sistemáticas.

Se concluye que la intervención educativa se asocia positivamente en disminuir significativamente las concentraciones de hemoglobina glicosilada. Serán necesarias investigaciones posteriores para definir las intervenciones más efectivas en reducir la HbAlc, hasta lograr el control metabólico recomendado $<7 \%$.

\section{Referencias}

1. Guyton A, Hall J. Treaty of medical physiology. Madrid: Elsevier Spain; 2006. p 949-50.

2. CEPAL. The process of demographic aging and its future 
trends. 1st ed. Collection Project documents Future scenarios in old age policies. Bogotá: 2008. p. 38.

3. Sapunar ZJ. Epidemiología de la diabetes mellitus en Chile. Rev Med Clin Las Condes 2016; 27: 146-51. DOI: 10.1016/j.rmclc.2016.04.003.

4. Carrasco F, Moreno M, Irribarra V, Rodríguez L, Martin MA, Alarcón A, et al. Evaluation of a pilot intervention program in adults with overweight or obesity, at risk of diabetes. Rev Med Chile [Internet] 2008; 136 (1): 13-21.

5. Churches R, Barutell L. Summary of the recommendations of the American Diabetes Association (ADA) 2014 for clinical practice in the management of diabetes mellitus. Depth; 2014. p. 2.

6. American Diabetes Association (ADA). Glycemic Targets. Diabetes Care January 2016; 39 (Suppl 1): S39-S46. DOI: 10.2337 / dc16-S008.

7. Reyes F, Pérez M, Alfonso E, Ramírez M, Jiménez Y. Current treatment of diabetes mellitus type 2. ccm [Internet] 2016; 20 (1): 98-121.

8. Aguilar Escobar J, Espinoza Dávila E. Evaluation of knowledge and dietary practice in type 2 diabetic patients. Gac Med Bol 2006; 29 (1): 17-20.

9. Tejada LM, Pastor M, Gutiérrez S. Effectiveness of an educational program in the control of patients with diabetes. Mexico, 2006. Invest Educ Enferm 2006; (24) 2: 48-53.

10. Fernández A, Abdala T. Self-care strategies in patients with type 2 diabetes mellitus. Rev Esp Med Quir 2012; 17 (2): 94-9.

11. Wagner EH, Austin BT, Davis C, Hindmarsh M, Schaefer J, Bonomi A. Improving Chronic Illness Care: Translating Evidence Into Action. Health Aff (Millwood)
2001; 20 (6): 64-78. doi: 10.1377 / hlthaff.20.6.64.

12. Pereira DA, Costa NM, Sousa AL, Jardim PC, Zanini CR. Effects of educational intervention on knowledge of the disease in patients with diabetes mellitus. Rev Lat Am Enfermagem 2012; 20 (3): 478-85. Available from: http://www.scielo.br/scielo.php?script=sci_arttext\&pi$\mathrm{d}=$ S0104-11692012000300008\&lng=en. http://dx.doi. org/10.1590/S0104-11692012000300008.

13. Figueroa-Suárez ME, Cruz-Toledo JE, Ortiz-Aguirre AR, Lagunes-Espinosa AL, Jiménez-Luna J, Rodríguez-Moctezuma JR. Lifestyle and metabolic control in diabetics of the DiabetIMSS program. Gac Med Mex 2014; 150 (1): 29-34.

14. Llamazares Iglesias O, Sastre Marcos J. Metabolic and cardiovascular risk factor control in a diabetic cohort. Four-year results. Endocrinol Nutr 2012; 12 (2): 117 24.

15. León-Mazón MA, Arujo-Mendoza G. Efficacy of the diabetes education program in clinical and biochemical parameters: Rev Med Inst Mex seguro Soc 2013; 51 (1): 74-9.

16. García-Huidobro D. Family focus in Primary Care: A proposal to improve the health of all. Rev Med Chile 2010; 138 (11): 1463-4. Available at: http:// www.scielo.cl/scielo.php?script=sci_arttext\&pid= S0034-98872010001200019\&lng=es. http://dx.doi. org/10.4067/S0034-98872010001200019.

17. García R, Suárez R. Education for people with diabetes mellitus in primary health care. Rev $\mathrm{Cu}-$ bana Endocrinol 2007; 18 (1): Available at: http:// scielo.sld.cu/scielo.php?script $=$ sci_arttext\&pid= S1561-29532007000100005\&lng=es. 